\title{
AN INJECTION MOULDING DEVICE, IMPROVING THE EXISTING INJECTION SYSTEM FOR ACRYLIC DENTURE FABRICATION
}

\author{
Kalina Georgieva, Metodi Abadjiev \\ Department of Prosthetic Dental Medicine and Orthodontics, Faculty of Dental Medicine, \\ Medical University of Varna
}

\section{ABSTRACT}

INTRODUCTION: The advantages of injection molding system (IMS) are precise adaptation of acrylic to master cast, continuous compensation of acrylic shrinkage, low porosity and better physical properties of dentures. Traumatic, toxic and allergic complications are reduced. The existing IMS produces only one denture at a time. Using standard capsules leads to waste of material. Removable partial acrylic dentures (RPAD) with wire clasps haven't been fabricated yet.

MATERIALS AND METHODS: The objective is to construct a device for serial injection fabrication of acrylic dentures optimizing both working time and cost of restoration. The device should:

* provide a precise adaptation of material to master cast

* reduce residual monomer

* fabricate a lot of dentures for a short time

* fabricate RPAD with wire clasps

* optimize the amount of material.

A prototype of the device is designed and constructed.

RESULTS: The new device is based on the existing IMS.

The following improvements are applied:

* The serial fabrication is achieved by dividing the injection and polymerization process by including a specially designed cap which is fixed on the flask after acrylic injection. It provides continuous pressure during the polymerization process. This allows fabrication of large number of dentures at the same time, injected by a single pneumatic cylinder.

* RPAD with wire clasps are fabricated by fixing the clasps with cold-cured acrylics and mechanical retention in the master mast.

* Capsules for multiple fabrication are used.

CONCLUSION: A serial injection of large number of dentures as well as separate processing of each of them is possible at a time. The waste of material is reduced. Fabrication of RPAD with wire clasps is achieved.

Keywords: acrylic dentures, injection moulding device

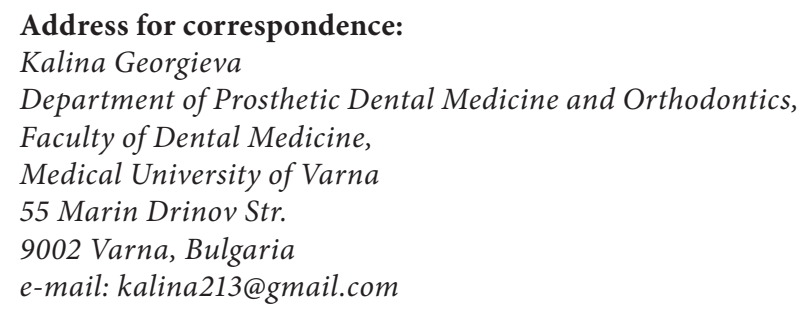

Received: December 7, 2014

Accepted: June 1, 2015 
An injection moulding device, improving the existing injection system for acrylic denture fabrication

\section{INTRODUCTION}

Total edentulism rates are getting lower in the USA (1) and other developed countries (2) but for Eastern Europe are still too high (3).

Prosthetic treatment with conventional complete dentures is a common decision for such cases because of the lower cost and approximately good clinical results such as satisfactory mastication, speech and esthetics. Besides financial problems, common health status and general medications could prevent other possible options such as implant treatment and overdentures. All these make conventional complete dentures pretty common prosthetic restorations for our country.

Conventional cuvette technique (compression moulding technique) is widely used for fabrication of acrylic complete dentures. But this technique has some significant disadvantages such as increased vertical dimensions $(4,5)$; higher porosity; spherical deformation; higher acrylic shrinkage; inaccurate fit of the material to the master cast and larger amount of residual monomer (6).

All these disadvantages are eliminated by using Injection moulding system.

In 1942 Pryor offered injection processing of poly methyl methacrylate in order to reduce acrylic shrinkage (7).

The injection moulding technique has proved its advantages during the years: precise adaptation of acrylics to the master cast, continuous compensation of acrylic shrinkage due to inflowing acrylic material and constant high pressure during the whole polymerization process, better physical properties of dentures, lower porosity, high degree of homogeneity and minimum level of residual monomer $(8,9,10,11)$.

Acrylic dentures using injection moulding of acrylic resin reduce traumatic, toxic and allergic complications. Patients' comfort is guaranteed by the precise fit of the acrylic dentures as well as minimum level of residual monomer and easy maintenance.

By now complete acrylic dentures and removable partial acrylic dentures with acrylic clasps has been fabricated by injection moulding technique. No evidence was found during our research about making removable partial acrylic dentures with wire wrought clasp.
Also only one denture could be made at a time with one device.

Last but not least, using standard capsules with poly methyl methacrylate resin

leads to waste of material because the quantity of the material is usually more than enough for injection of one denture but not enough for two dentures.

Our purpose is to offer some improvements in the existing devices for injection moulding of acrylic resin in order to expand the area of appliance- fabricating of removable partial acrylic dentures with wire wrought clasps. Also we'd like to optimize the laboratory working time and the quantity of the necessary material by introducing an option for serial injection moulding with one and the same device and different capsule sizes with different quantity of acrylic resin enough for fabrication of several dentures during the serial usage mode of the device.

The new improved device should:

* provide a precise adaptation of material to the master cast

* reduce the residual monomer

* fabricate a lot of dentures for a short time

* fabricate partial acrylic dentures with wrought wire clasps

* optimize the amount of necessary material

\section{MATERIALS AND METHODS}

A prototype of the device is based on the existing systems.

One of them is the injection system IVOCAP by Ivoclar Vivadent (Fig. 1).

The wax denture pattern is prepared for processing by investing in a dental flask and dewaxing. Inside the flask a mould space is formed for acrylic to fill in.

A standard capsule containing powder and liquid of acrylic resin is mixed in a separate device.

A pressure apparatus, a capsule with methyl methacrylate and a flask are fixed together using two vise shanks, a safety loop and a clamping frame. The injection of the acrylic resin (methyl methacrylate) into the flask is done by the pressure apparatus under pressure of 6 bars. The pressure stays constant during the whole curing process in order to compensate the acrylic shrinkage by injection of new acrylic amounts. Both pressure apparatus and flask are oc-

Scripta Scientifica Medicinae Dentalis, vol. 1, №1, 2015, 31-37 Copyright (c) Medical University of Varna 

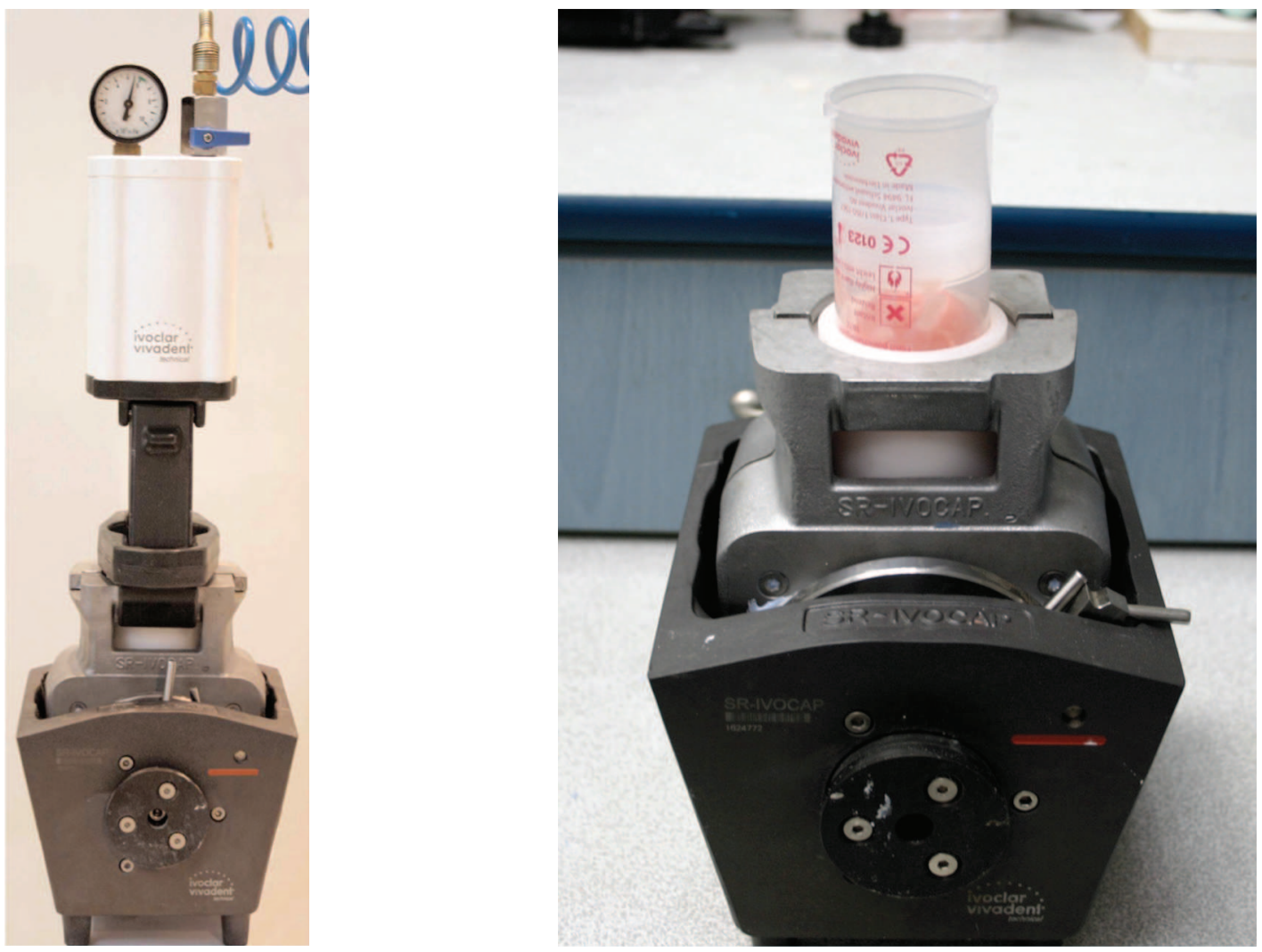

Fig. 1. The injection system IVOCAP by Ivoclar Vivadent

cupied during the polymerization process and cooling. After that the pressure apparatus and flask are separated. The processed denture is deflasked, finished and polished.

The injection process in the improved system (Fig. 2) repeats the existing one. The denture pattern is invested in the two halves of the flask (8). After dewaxing, inside the flask (8) a mould space, corresponding to the acrylic part of the denture, is formed.

In the upper part of the flask (8) the capsule plunger (7) for standard capsules or capsules with different volume of acrylic resin (used for serial fabrication of 2, 4 or 6 dentures) is situated.

The flask is attached to the pneumatic cylinder (1) through two vise shanks (5) and a safety loop (6). This is the injection unit.

The pneumatic cylinder (1) (type NWT 50.80) has a manometer (2) and a control part with locking valve (12).

The locking valve opens or closes the high pressure outlet (11). The injection of the acrylic resin into the mould space of the flask is done by the extrusion piston (4) under pressure 6 bars (the locking valve is opened).

The locking valve is closed so the pressure supply is over. The extrusion piston goes up and the pneumatic cylinder and the flask are separated by releasing the vise shanks and safety loop.

A sealed lid (9), shown in details on Fig. 3 and Fig. 4, is fixed on the flask (8) through two vise shanks (5) and a safety loop (6) (Fig. 5). The lid could resist 10 bar pressure which is necessary because a high pressure outlet (11) is attached to the sealed lid in order to supply pressure 6 bars during the polymerization process instead of the pneumatic cylinder.

Fig. 6 shows the polymerization unit, consisting of the flask (8), the sealed lid (9) and the clamping frame (10).

The polymerization unit is put in water bath for curing the acrylic resin (35 min from the beginning of boiling) and after that in cold water bath for the cooling process ( 30 minutes). Then the acrylic denture is deflasked so the flask, the sealed lid 


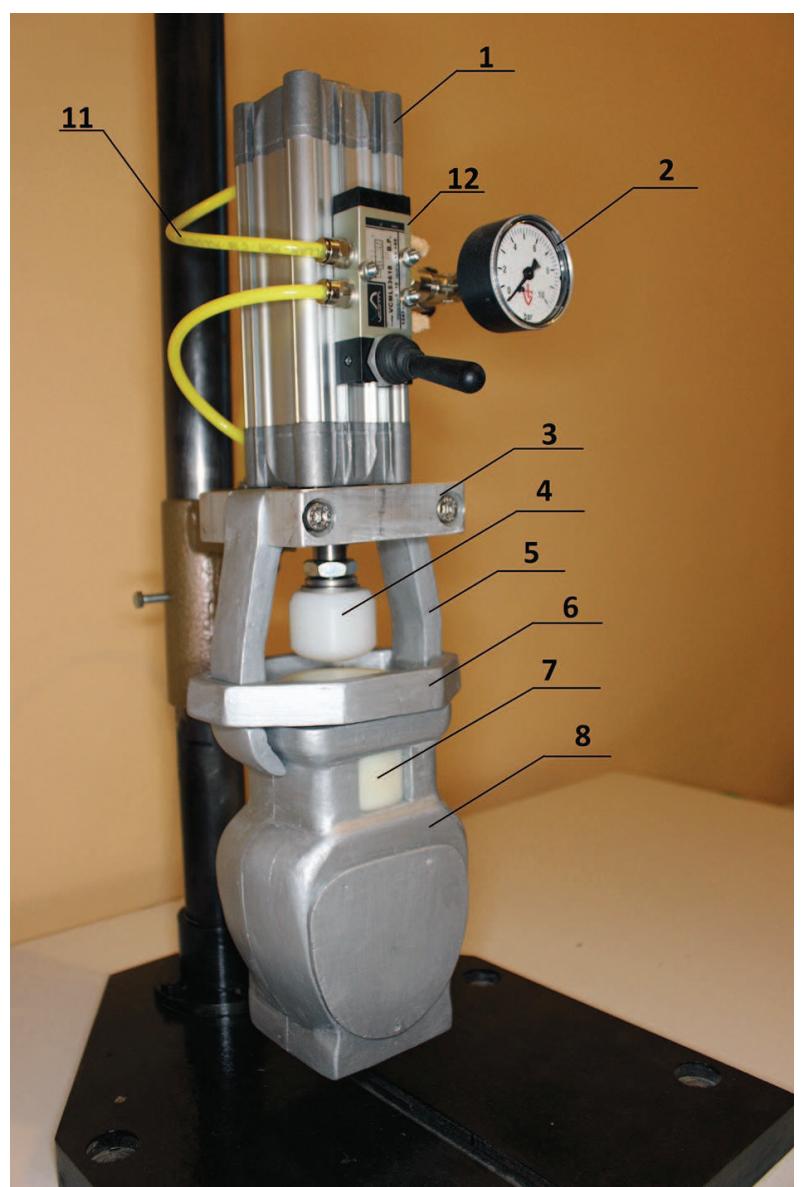

Fig. 2. The injection unit of the improved system: 1-pneumatic cylinder, 2-manometer, 3-base of vise shanks, 4 extrusion piston, 5 - vise shank, 6 - safety loop, 7 - capsule holder, 8 -flask, 11 - high pressure outlet, 12 - control part and locking valve of pneumatic cylinder

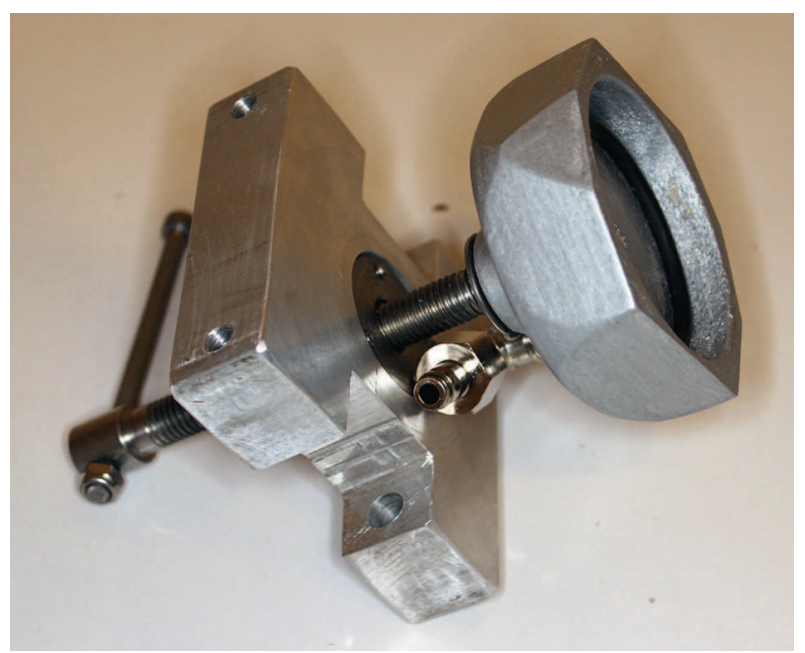

Fig. 3. A sealed lid - side view

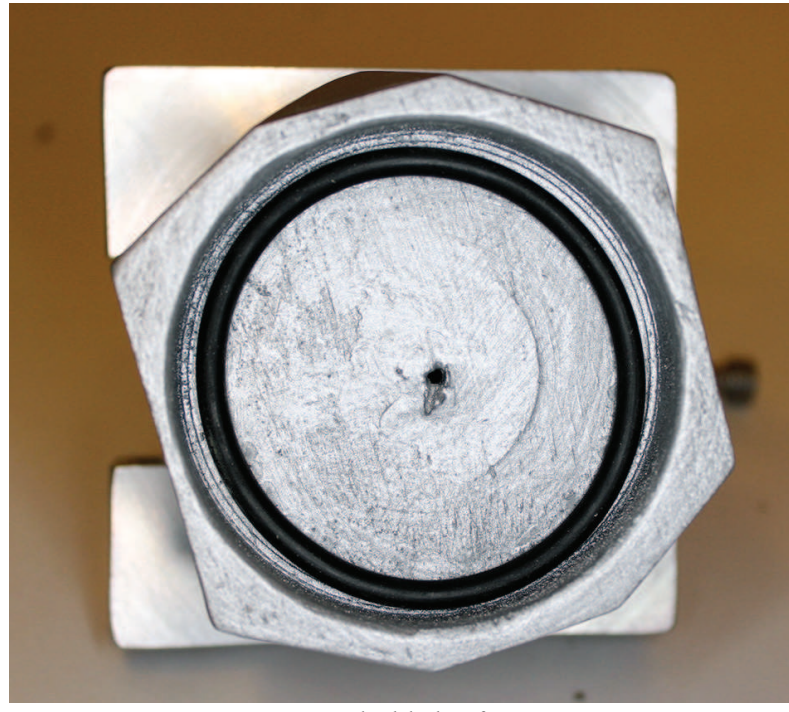

Fig. 4. A sealed lid - front view

and the clamping frame are ready for a new denture fabrication.

During all this time the pneumatic cylinder is free for acrylic injection of other flasks. The injection moulding takes 5 minutes which means that a single pneumatic cylinder could inject at least 15 flasks during the curing and cooling process of the first flask. This improves the efficiency of denture fabrication and reduces the necessary time.

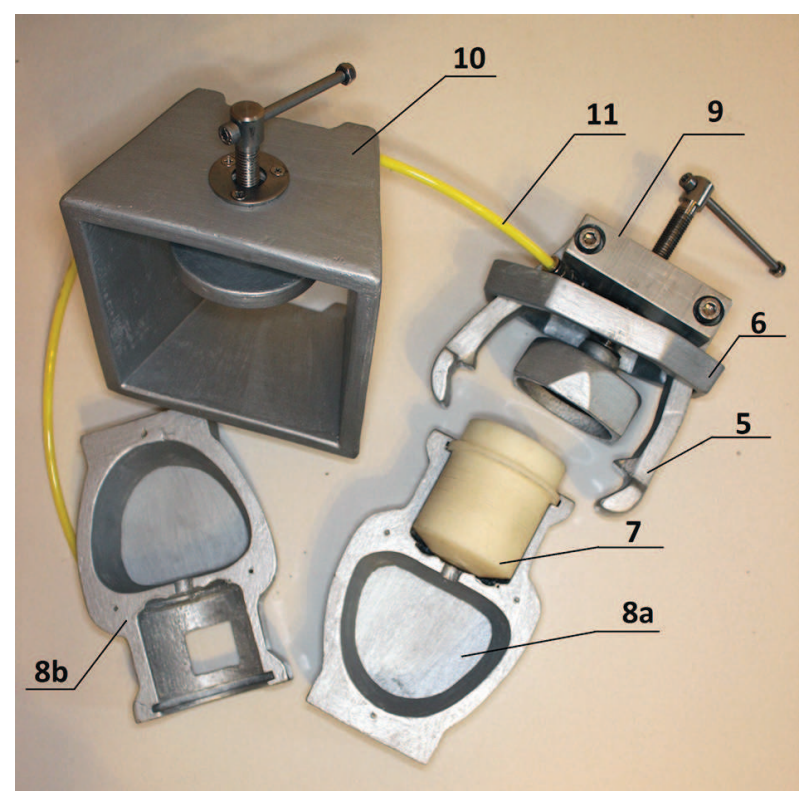

Fig. 5. Parts of polymerization unit: 5 - vise shank, 6 safety loop, 7 - capsule holder, $8 a$ and $8 b$ - two halves of flask, 9 - sealed lid, 10 - clamping frame, 11 - high pressure outlet 


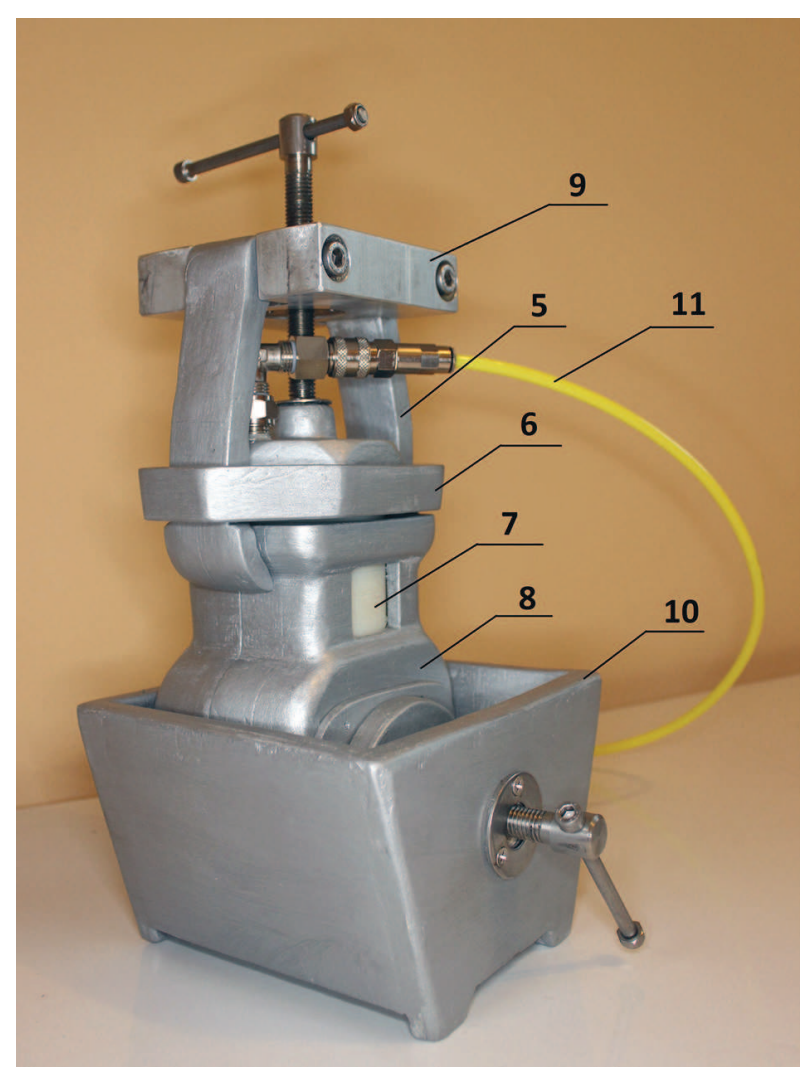

Fig. 6. The polymerization unit: 5 - vise shank, 6 - safety loop, 7 -capsule holder, 8 - flask, 9 - sealed lid, 10 clamping frame, 11 - high pressure outlet

The capsules consist of monomer and polymer of acrylic resin (methyl methacrylate) in ratio recommended by the producer. The acrylic resin is mixed in a separate device. The diameter of its capsule hold-

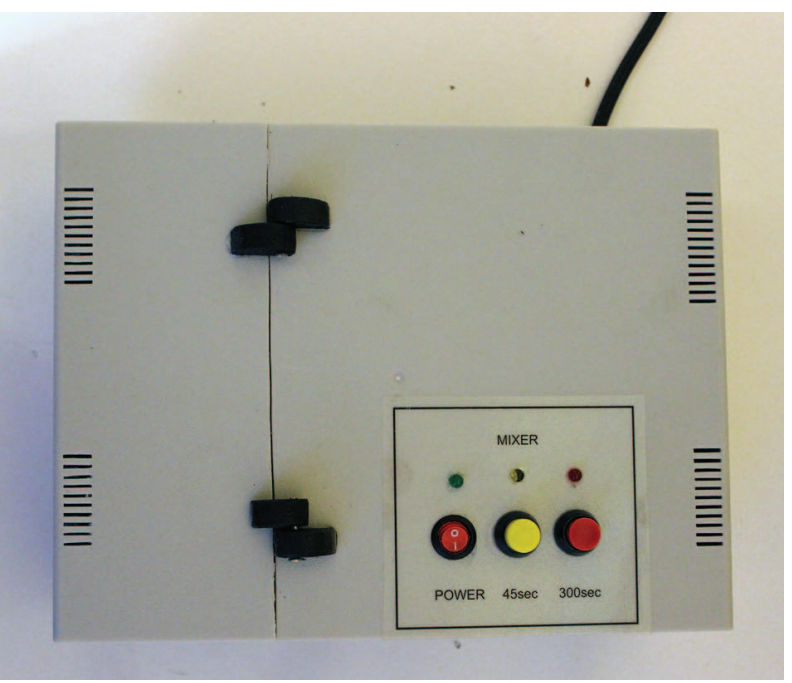

er is standard so it could be used with standard capsules and capsules with different volume due to different length of the capsules suitable for serial fabrication of 2, 4 or 6 dentures. The mixing is done by reciprocating and rotating movements for 45 seconds. After 300 seconds the mixing device turns off automatically (Fig. 7).

The fabrication of partial acrylic dentures with wire wrought clasps is possible if dentures are invested in flasks in a particular way.

A mechanical retention is formed in the dental stone of the master cast (Fig. 8.1). The retentive clasp arm is fixed to the master cast by self-curing transparent acrylic resin (Fig. 8.2). After that the wax pattern of the partial denture is finished on the master cast (Fig. 8.3). The clasp arm located on the abutment tooth could be fixed in two ways.

The first one is by removing the dental stone tooth, forming a mechanical retention in the dental stone and fixing with self-curing acrylic resin (Fig. 8.4 and Fig. 8.5.). The second way is by covering the master cast and the clasp arm with dental stone (except for the artificial teeth and wax).

In these ways the claps is stable on the master cast and there is no risk of displacement during injection moulding.

The denture is invested in a flask (Fig. 8.6). After injection moulding, polymerization process, cooling, finishing and polishing the partial denture with wire wrought clasps is ready (Fig. 8.7).

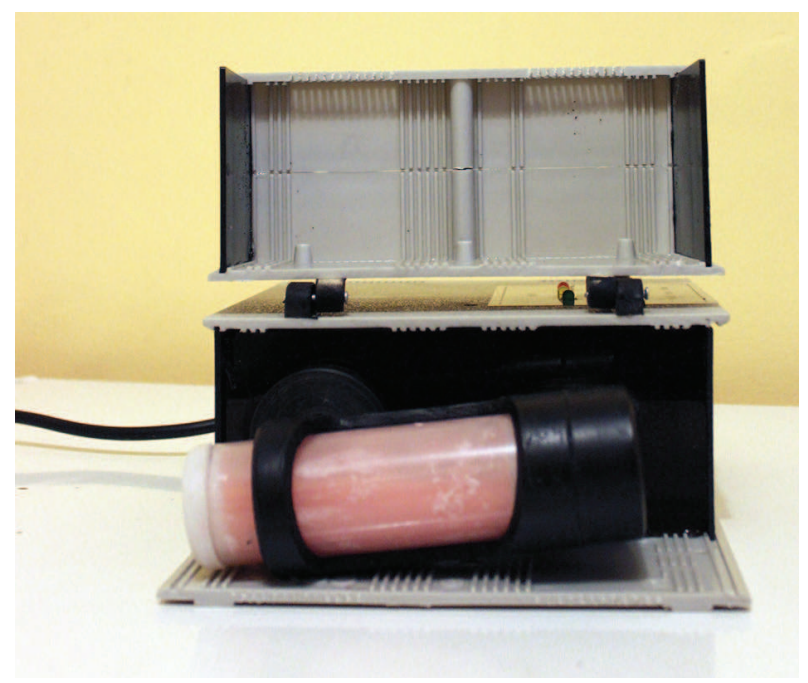

Fig. 7. Mixing device 


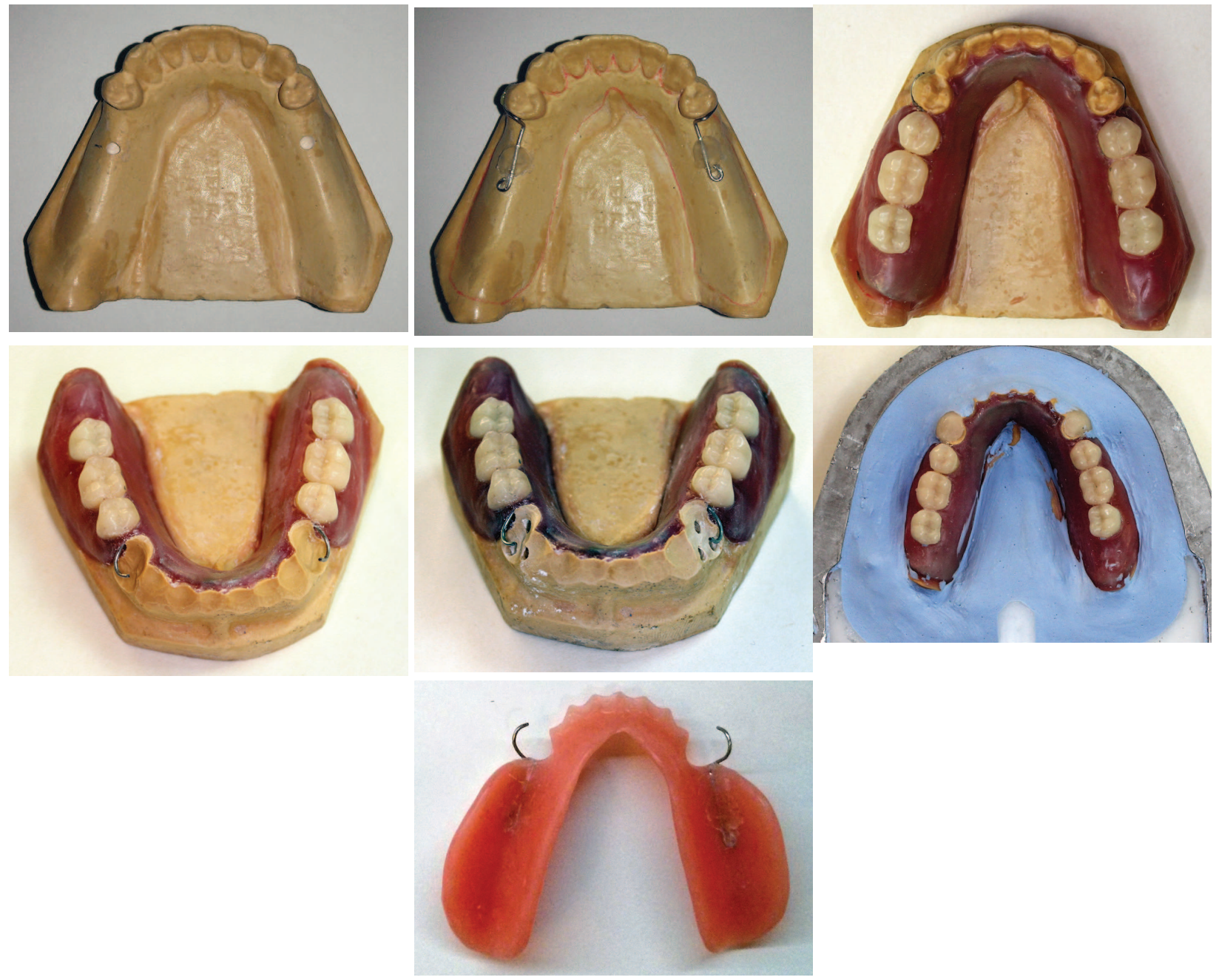

Fig. 8. Fabrication process of partial acrylic dentures with wire wrought clasps

\section{RESULTS}

The serial fabrication of acrylic dentures is achieved by dividing the injection and polymerization process by including a specially designed sealed lid which is fixed on the flask after acrylic injection. It provides continuous pressure during the rest of the polymerization process. This allows fabrication of larger number of dentures - at least 15, injected one by one by a single pneumatic cylinder (the injection unit) and 15 polymerization units for finishing the polymerization process of the acrylic dentures in water bath especially when capsules with different volume are used. In comparison the existing system could be used for fabrication of only one denture at the same time. This makes the improved sys- tem more efficient as far as working time and material are concerned.

The standard capsules could also be used with the improved device but we also offer capsules with different volume for serial fabrications of 2, 4 or 6 dentures. The diameter of the capsule is one and the same but the different length of the capsule provides different quantity of acrylic resin.

Removable partial acrylic dentures with wire wrought clasps are fabricated by fixing the clasps with cold-cured transparent acrylic resin and mechanical retentions in the master cast. 


\section{DISCUSSION}

The injection moulding system is a more accurate method for processing dentures compared to the standard compression moulding technique.

The fabrication process of complete dentures is optimized by dividing the injection and polymerization process by introducing a specially designed cap (a sealed lid) which is fixed on the flask after acrylic injection for keeping constant high pressure during polymerization process.

At least 15 dentures could be processed (injected and cured) with the improved system compared to only one denture with the existing system for one and the same time.

The waste of material is reduced by $30 \%$ by capsules with different volume due to the number of injected dentures. The application of the system is expanded - partial acrylic dentures with wire wrought clasps could be made by simple fixing of the clasp with self-cured resin and mechanical retentions in the master cast.

\section{CONCLUSIONS:}

The introduced improvements of the existing injection moulding system solve some problems in dental laboratory work. The working time and the cost of the prosthetic restorations are reduced. Another type of partial dentures - partial acrylic dentures with wire wrought clasps, could be fabricated. This would be very efficient and useful in bigger dental laboratories such as those in dental faculties where dental students fabricate removable dentures on their own.

\section{REFERENCES:}

1. Wu B, Liang J, Plassman BL, Remle C, Luo X. Edentulism trends among middle-aged and older adults in the United States: comparison of five ra$\mathrm{cial} / \mathrm{ethnic}$ groups. Community Dent Oral Epidemiol. 2012 Jun; 40(3):288.

2. Mojon P, Thomason JM, Walls AW. The impact of falling rates of edentulism. The International Journal of Prosthodontics. 2004;17(4):434-440.

3. Petersen PE, Kandelman D, Arpin S, Ogawa H. Global oral health of older people - Call for public health action. Community Dental Health. 2010; 27(Suppl 2):257-268.
4. Mutti NM, Pazzini LI. Horizontal fit of flasks as a factor in complete denture disarticulation. The Journal of Prosthetic Dentistry. 1974;32(4):448-452.

5. Matsuda R, Yoneyama Y, Morokuma M, Ohkubo $\mathrm{Ch}$. The influence of vertical dimension of occlusion changes on the electroencephalograms of complete denture wearers. Journal of Prosthodontic Research. 2014;58:121-126.

6. Dogan A, Bek B, Çevik NN, Usanmaz A. The effect of preparation conditions of acrylic denture base materials on the level of residual monomer, mechanical properties and water absorption. Journal of Dentistry. 1995;23(5):313-318.

7. Pryor WJ. Injection moulding of plastics for dentures. J Am Dent Assoc. 1942;29:1400-1408.

8. Anderson GC, Schulte JK, Arnold TG. Dimensional stability of injection and conventional processing of denture base acrylic resin. Dental Technology. 2nd ed. 1988;60(3):394.

9. Nogueira SS, Ogle RE, Davis EL. Comparison of accuracy between compression- and injectionmoulded complete dentures. The Journal of Prosthetic Dentistry. 1999;82(3):291-300.

10. Shadi EB, Ludwig K, Shamran A et al, Linear and volumetric dimensional changes of injectionmoulded PMMA denture base resins. Dental materials. 2013;29:1091-1097.

11. Garfunkel E. Evaluation of dimensional changes in complete dentures processed by injection-pressing and the pack-and-press technique. J Prosthet Dent. 1983;50(6):757-761. 